\title{
Erratum
}

\section{Phase Space Bounds for Quantum Mechanics on a Compact Lie Group}

Brian C. Hall

Department of Mathematics, McMaster University, Hamilton, Ontario, L8S 4K1, Canada

Received: 30 September 1997 / Accepted: 30 September 1997

Commun. Math. Phys. 184, no 1, 233-250 (1997)

Three times on p. 238 the symbol $\gamma$ appears. This symbol should be $\dot{\gamma}$, indicating the time derivative of $\gamma$.

Communicated by D. Brydges 